\title{
Zwischen der wörtlichen und der phraseologischen Bedeutung. Zur Semantik der metaphorisch motivierten Idiome aus der Perspektive der Kognitiven Grammatik
}

\author{
Anna Sulikowska (Stettin)
}

\begin{abstract}
The aim of the article is to describe the metaphoric motivated German idiom jmdn. in die Zange nehmen in the scope of Ronald Langacker's Cognitive Grammar. The author begins her analysis with a description of the conceptual content of the lexical unit ZANGE in its literal meaning to establish the amount and art of domains, which are activated within the unit and their influence on the unit's conceptualization in the metaphoric and phraseological meaning.
\end{abstract}

\section{$1 \quad$ Einleitung}

Die Erfassung der Bedeutung einer sprachlichen Einheit gehört zu den wichtigsten und zugleich schwierigsten Aufgaben der Lexikographie. Ihre vollkommene Ausführung ist, wie die neueren kognitiv ausgerichteten semantischen Theorien beweisen, unerreichbar, da die Bedeutungen nicht mehr als statische, sondern als dynamische, sich bei jedem Gebrauch von neuem konstituierende Entitäten betrachtet werden. Somit können selbst die Konzeptualisierungen von Konkreta (die wegen der deutlichen Referenz als relativ leicht zu beschreibende Sprachzeichen gelten) wesentliche Differenzen aufweisen, die vom Kontext, dem individuellen Weltwissen und von den Emotionen abhängen. Noch schwieriger ist die Erfassung der Bedeutung von Phraseologismen, die des Öfteren über zwei Lesarten, eine wörtliche und eine phraseologische, verfügen.

Im folgenden Beitrag wird ein Versuch vorgenommen, am Beispiel eines metaphorisch motivierten Idioms mit disjunktiver Lesart ${ }^{1}: j m d n$. in die Zange nehmen die Komplexität der Idiom-Semantik aufzuzeigen. Als theoretische Grundlage der Analyse wird die Theorie der Kognitiven Grammatik (KG) von Ronald Langacker herangezogen, die in Anlehnung an Cognitive Grammar. A Basic Introduction (2008, poln. 2009) dargestellt wird. Wir gehen von der Beschreibung der ZANGE und ihren möglichen Konzeptualisierungen in der wörtlichen Lesart aus, schildern dann kurz die Metapher-Problematik, um schließlich die Frage aufzu-

\footnotetext{
1 Als Phraseologismen mit einer disjunktiver Lesart bezeichnet Burger (2010: 63) Phraseologismen mit zwei Lesarten, in denen die wörtliche und phraseologische (lexikalisierte) Lesart nicht in den gleichen Kontexten oder Kommunikationssituationen vorkommen können.
} 
werfen, wie die Zange als Hauptkonstituente einer zusammengesetzten Spracheinheit konzeptualisiert wird, die zugleich metaphorisch und lexikalisiert ist.

\section{Die Auffassung der Bedeutung in der KG}

Die Grundlage der Theorie von R. Langacker bildet die Annahme, dass die Bedeutungen mit den Konzeptualisierungen gleichzusetzten $\operatorname{sind}^{2}$ (vgl. Langacker 2008: 30). Die Konzeptualisierungen weisen dabei einen dynamischen Charakter auf, sind eng an den Sprachgebrauch gebunden, in dem sie sich jedes Mal von neuem konstituieren. Außer den dynamischen, offenen, flexiblen und individuellen Konzeptualisierungen müssen die Sprachteilhaber über eine gemeinsame vage Bedeutungskonzeption verfügen, um kommunizieren zu können:

Speakers must have some preconception of what the words they use are normally expected to mean. Otherwise the meanings negotiated would be completely random, and cat would have no greater likelihood of meaning 'feline' than 'walnut', 'book', or 'through'. While everything may be negotiable, something has to be learned and conventionalized as a basis for negotiation.

(ebd.: 30)

Somit werden in Langackers Theorie auch stabilere semantische Strukturen vorausgesetzt, die auf der Grundlage der Operationen der Schematisierung und der Kategorisierung aus den Sprachgebrauchsereignissen (und den mit ihnen zusammenhängenden Konzeptualisierungen) entstehen. Im Fokus des Interesses von der KG befinden sich die Konzeptualisierungen, die wiederum aus zwei Bestandteilen, dem konzeptuellen Inhalt und der Interpretation (constru$a l)$, aufgebaut sind.

\subsection{Der konzeptuelle Inhalt in der enzyklopädischen Semantik}

Eine weitere wichtige Voraussetzung Langackers Theorie bildet der Verzicht auf die Abgrenzung des Weltwissens vom Sprachwissen: In der im Rahmen der KG vertretenen enzyklopädischen Semantik ergibt sich die lexikalische Bedeutung aus dem mentalen Zugang zu einer offenen Menge von dem sich auf ein Objekt/Phänomen beziehenden Wissen, das sowohl sprachliche als auch kulturell geprägte und enzyklopädische Elemente umfasst.

\section{Encyclopedic Semantics}

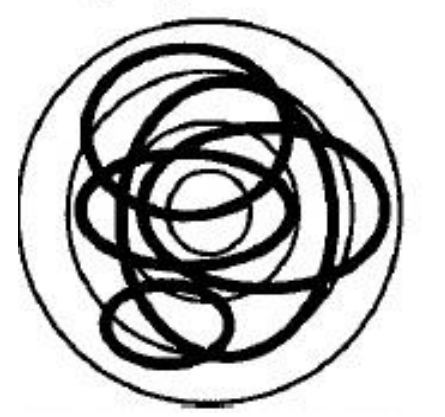

Abb. 1: Lexikalische Bedeutung als mentaler Zugang zu Wissensdomänen in der enzyklopädischen Semantik (Langacker 2008: 39)

Dieses Wissen ist auf dem Bild in Form von Ellipsen dargestellt, die die Domänen symbolisieren. Unter dem Begriff Domäne versteht Langacker (ebd.: 44, 51) kohärente und geordnete

2 "Meaning is not identified with concepts but with conceptualization" (Langacker 2008: 30). 
konzeptuelle Inhalte oder Erfahrungssphären, die durch einen sprachlichen Ausdruck evoziert werden $^{3}$. Nicht allen Domänen kommt der gleiche Status zu: Während die Basisdomänen (wie beispielshalber die Domänen des Raumes, der Zeit, der Farbe, der Tonhöhe, der Temperatur u. a.) kognitiv nicht reduzierbar sind (vgl. ebd.: 44), sind zahlreiche andere Domänen "non basic" (nicht-elementar) und unterscheiden sich sehr wesentlich in ihrer Größe und in der Komplexität ihrer inneren Struktur. Sie umfassen alle einem Sprecher zu einem Sachverhalt zugänglichen Informationen, weswegen sie individuelle Züge aufweisen und wenigstens zum Teil durch die Einzigartigkeit eines Individuums und seiner Erfahrungen geprägt sind. Darüber hinaus liegen die Domänen auf konzentrischen Kreisen, die die Prototypikalität versinnbildlichen. Je näher dem Zentrum sich eine Domäne befindet, desto größer ist die Wahrscheinlichkeit, mit der sie beim Gebrauch eines Wortes mental zugänglich wird (vgl. Langacker 2008: 48): Der im Zentrum befindliche Kreis beinhaltet demnach Informationen, die bei beinahe jeder Aktivierung einer sprachlichen Einheit aufgerufen werden, das in weiteren Kreisen gespeicherte Wissen betätigt man demgegenüber nur in besonderen Kontexten (vgl. ebd.: 39).

Somit könnte das Konzept der ZANGE beispielshalber folgendermaßen beschrieben werden: Die Basisdomäne des Raumes - ZANGE als ein Objekt im Raum (Domäne 1) - könnte um weitere, nicht-elementare Domänen erweitert werden, die verschiedene enzyklopädische Informationen beinhalten. In der perzeptuellen Domäne der Form (Domäne 2) könnte die Zange als ein zweischenkliges, der Schere ähnelndes Werkzeug dargestellt werden, das aus zwei durch einen Drehbolzen verbundenen Greifbacken oder Schneiden besteht. Im Gegensatz zu der Schere gleiten die Bearbeitungsflächen aber nicht aneinander vorbei, sondern lassen einen Gegenstand fixieren oder bearbeiten. Typischerweise sind die Schenkel länger als die Greifbacken, relevant ist auch das Wissen, dass die Zange normalerweise von einer Person bedient wird (Domäne 3). Die Zange funktioniert des Weiteren nach dem Hebelprinzip, weswegen die Kräfte, die mit diesem Gegenstand erzeugt werden, groß sind (Domäne 4). Als ein Mehrzweckwerkzeug dürfte die Zange ebenfalls viele funktionale Domänen aktivieren: Zange macht damit in der Funktion (Domäne 5) ein populäres Werkzeug zum präzisen, sicheren Festhalten von Objekten (so können beispielshalber die Nägel beim Schlagen mit der Zange festgehalten werden). Die Zangen mit scharf geschliffenen Bearbeitungsflächen (Eckzangen, Kneifzeigen) können zum Abschneiden gebraucht werden (Domäne 6), andere zum Biegen von Gegenständen (Domäne 7). Die Zange, z. B. Flachzange, kann ebenfalls zum Quetschen von Objekten eingesetzt werden (Domäne 8). Darüber hinaus findet die Zange heutzutage Verwendung in der Medizin (Domäne 9), wo sie wenigstens von Laien mit unangenehmen, blutigen Eingriffen assoziiert wird (Zahnextraktion, Geburtshilfe). In der Domäne (10) wird sie als ein Instrument zum indirekten Anfassen, sauberen und hygienischen Servieren von Lebensmitteln im Haushalt und im Handel (Zuckerzange, Gebäck-Zange, Salatzange usw.) ${ }^{4}$ konzeptualisiert. In der nächsten Domäne (11) könnte das Wissen über die Zange als ein Greifwerkzeug von Tieren gesammelt sein.

\footnotetext{
${ }^{3}$ Vgl. auch die Definition von Taylor (2007: 528): Domänen sind geordnete mentale Vorstellungs- oder Erfahrungsbereiche, die bei der Bestimmung der Bedeutung von Relevanz sind.

4 Diese Assoziation mag einen entscheidenden Beitrag zur Bedeutungskonstituierung des Phraseologismus jmdn./etw. nicht mal mit der Zange anfassen mögen leisten.
} 
Die beschriebenen Domänen mögen einem durchschnittlichen erwachsenen Sprachteilhaber in verschiedenem Ausmaß zugänglich sein, das ihnen zu Grunde liegende Wissen wurde durch die Beobachtung bzw. Handhabung der Zange in alltäglichen Situationen erworben. Eine andere Provenienz haben zwei weitere Domänen, die, auf das historische Wissen zurückgreifend, den meisten Sprachteilhaber heutzutage nicht aus täglicher Erfahrung, sondern eher aus Schulbildung, Reisen usw. bekannt sein könnten. Zum einen spielte die Zange eine bedeutende Rolle im Altertum und Mittelalter: Als ein zum Greifen und Bewegen von heißen Gegenständen dienendes Werkzeug, mit dem ein Schmied glühendes Eisen sicher festhalten und über längere Zeit bearbeiten konnte, gehörte sie zu den zentralen Attributen der Schmiedekunst, wovon z. B. die Abbildungen des griechischen Schmiede- und Feuergottes Hephaistos zeugen (Domäne 12) ${ }^{5}$. Zu dieser Zeit wurde sie auch als ein Folter- und Hinrichtungswerkzug sowie ein Werkzeug zur Vollstreckung von Körper- bzw. Verstümmelungsstrafen gebraucht (Domäne 13): "Die Hinrichtungen waren so grausam, wie die Foltern: Zwicken mit glühenden Zangen, Riemen aus dem Rücken schneiden, Viertheilen, Rädern, Händeabhacken, Herzausschneiden und ums Gesicht schlagen waren an der Tagesordnung" (Menzel 1834: 792, zit. nach www.redensarten.net/Zange.html). Dieses historische Wissen mag den meisten erwachsenen Sprachbenutzern zugänglich sein, weswegen sie sich bewusst sind, dass das Kneifen, Zwicken bzw. Festhalten mit der Zange sehr schmerzhaft und unangenehm sein müsste.

\subsection{Die Interpretation (construal) in der KG}

Die besprochenen Wissensdomänen bilden den konzeptuellen Inhalt (conceptual content), der dennoch lediglich den Ausgangspunkt der Bedeutungskonstituierung ausmacht ${ }^{6}$. Im realen Sprachgebrauch haben wir nämlich nicht mit den einzelnen Konzepten, sondern mit kontextbedingten Äußerungen zu tun. Die Konzeptualisierungen der Zange weisen in den folgenden Gebrauchsbelegen ${ }^{7}$ deutliche Differenzen auf:

(1) In der Luft hängt ein Surren vornehm gesenkter, fröhlicher Stimmen, vermischt mit dem Klirren von zierlichem Silberbesteck, das sachte an Porzellantassen mit königsblauen Blümchenrändern schlägt. Die Kellnerin im schwarzen Etuikleid fischt mit einer goldenen Zange [Hervorhebung: A.S.] zartes Gebäck daraus, Scones und Cakes und Gingerbread. Isn't it lovely?

(Die Zeit 24.07.08, zit. nach DWDS, s. v. Zange)

(2) Ein Vorort im Süden Teherans. Der Lehrer M. sitzt am Rand einer Baustelle auf einer alten Wolldecke zwischen Kabeln und Zangen [Hervorhebung: A.S.]. Dies ist sein Zweitjob, er baut die Elektrik für Straßenlaternen zusammen.

(Die Zeit 26.06.09, zit. nach DWDS, s. v. Zange)

\footnotetext{
${ }^{5}$ Damit wird im Duden (Redewendungen) (2011: 895) die Herkunft des Idioms erklärt: "Diese und die folgende Wendung jmdn., etw. nicht mit der Zange/Beißzange/Feuerzange/Kneifzange/Kohlenzange anfassen [mögen/wollen] bezogen sich ursprünglich auf das Schmieden, bei dem der Schmied das glühende Eisen mit der Zange festhält."

6 "Linguistic meaning involves both conceptual content and the construal imposed on that content" (Langacker 2008: 44).

${ }^{7}$ Alle Gebrauchsgelege wurden dem DWDS-Korpus entnommen. Suchanfragen: "Zange", "in\&\&@die\&\& Zange", "in\&\&@die\&\&Zange\&\&nehmen" [12.01.14].
}

ISSN 1615-3014 
Während bei der Bedeutungskonstituierung der Spracheinheit ZANGE in der ersten Äußerung die Domänen eines vornehmen Cafés, der höflichen Bedienung, des hygienischen Servierens u. ä. ihren Anteil haben, werden bei der zweiten Aussage die Domänen der beruflichen Tätigkeit eines Elektroinstallateurs, der Elektrizität, der Stromleitung aufgerufen. Die Domänen, die zur Bildung einer Bedeutung in einer bestimmten Äußerung beitragen, werden Domänenmatrix ${ }^{8}$ genannt, während man das in der Domänenmatrix gesammelte Wissen mit einer Szene vergleichen kann. Genauso wichtig wie die Szene ist bei der Bedeutungskonstituierung auch die weitgehend durch den Kontext bestimmte Betrachtungsperspektive: der Abstand zu der Szene, das Interesse des Betrachters, der seine Aufmerksamkeit auf bestimmte Elemente des Ganzen richtet, der Gesichtswinkel (vgl. ebd.: 55). Diesen Aspekt der Bedeutung nennt Langacker construal (Interpretation, Deutung) und verweist darauf, dass er aufs Engste mit dem Kontext zusammenhängt: Von allen zugänglichen Domänen werden nur diejenigen aktiviert, die zur Rezeption einer sprachlichen Einheit in einem konkreten Kontext nötig sind. Aus den aktivierten Domänen konstituiert sich dann ein Überlappungsbereich (scope), innerhalb dessen bestimmte Strukturen besonders hervorgehoben werden. Den Überlappungsbereich aller aktivierten Domänen nennt Langacker Basis, während besonders hervorgehobene Strukturen, auf denen die Aufmerksamkeit fokussiert ist, als Profile bezeichnet werden.

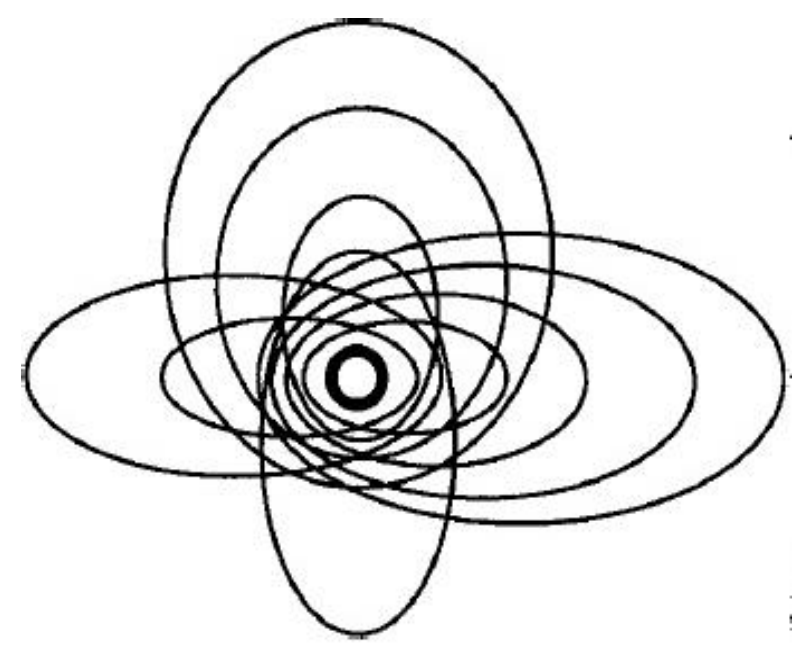

Abb. 2: Beziehung zwischen den Domänen (als Ellipsen dargestellt) und dem Profil (als umrundeter Kreis schematisch dargestellt) in der Konzeptualisierung (Langacker 2008: 48)

Langackers Bedeutungsauffassung hebt somit hervor, dass die Bedeutungen hauptsächlich als ein Produkt einer kreativen Konstruktionsaktivität des Individuums betrachtet werden müssen. Sowohl der konzeptuelle Inhalt als auch der Prozess seiner Interpretation (construal) sind wenigstens teilweise individuell und dynamisch, weswegen auch die Konzeptualisierungen vorübergehend, unscharf und dynamisch sein müssen. Diese Eigenschaften der Konzeptualisierungen, die Langacker überzeugend am Beispiel des Gebrauch von einem primären semiotischen Sprachzeichen (vgl. die Beschreibung der Bedeutung des Nomens glass von Lang-

\footnotetext{
8 Es ist dennoch hervorzuheben, dass die Domänenmatrix bei verschiedenen Sprachteilhabern bis zu einem gewissen Grade individuell und arbiträr ist: "We should not expect to arrive at any exhaustive list of the domains in a matrix or any unique way to divide an expression's content among them - how many domains we recognize, and which ones, depends on our purpose and to some extent is arbitrary. The important thing is to recognize the diverse and multifaceted nature of the conceptual content an expression evokes" (Langacker 2008: 44).
} 
acker 2008: 48-51) veranschaulicht, gelten desto mehr für sekundäre Sprachzeichen, zu denen metaphorisch motivierte Idiome gehören.

\section{Die Metapher in der KG}

Obwohl sich die Metapher-Problematik nicht im Zentrum des Interesses der KG befindet, nimmt Langacker an einigen Stellen seiner Einführung ansatzweise Stellung zur Rolle der Metapher und ihrem Status in der Sprache (2008: 36, 51, 58, 215). Seine Ansichten zur Natur der Metapher lassen sich dabei relativ einfach mit den im Rahmen der Kognitiven Linguistik vertretenen Metapher-Theorien - der Theorie der Konzeptuellen Metapher (CTM, Conceptual Theory of Metaphor) von Lakoff und Johnson (1980) und der Theorie von mentalen Räumen (mental spaces) von Fauconnier $(1985,1997)$ - in Einklang bringen, auch wenn Langacker die Metapher aus einer anderen Perspektive und mit einer anderen Zielsetzung beschreibt. ${ }^{9}$

Die Metapher wird im Rahmen der CTM mit der Annahme von zwei Domänen - der Ausgangsdomäne (source domain) und der Zieldomäne (target domain) - erklärt, zwischen denen feste Beziehungen (konzeptuelles Mapping) bestehen. $\mathrm{Zu}$ den wichtigen Erkenntnissen der CTM gehört die Voraussetzung, dass der Ausgangsbereich in der Sphäre der direkten menschlichen Erfahrung, der alltäglichen Tätigkeiten angesiedelt ist, während der Zielbereich eine abstraktere Sphäre darstellt (vgl. Lakoff 1993: 245). Domänen-ähnliche konzeptuelle Strukturen werden ebenfalls in der Theorie der mentalen Räume angenommen. Während aber die Domänen in der KG als geordnete, wenigstens teilweise etablierte Wissensstrukturen verstanden werden, beziehen sich mentale Räume auf semantische Repräsentationsformate für konkrete Äußerungsbedeutungen, die an den Prozessen der konzeptuellen Verschmelzung (blending) maßgeblich beteiligt sind ${ }^{10}$ (vgl. Ziem 2014: 3). Da die Domänen in der KG in ihrer internen Komplexität wesentlich variieren und ihre Beziehungen in der Domänenmatrix auch unterschiedlicher Natur sind, sind sie als Terminus ausreichend geräumig, um als ein Überbegriff für die Ausgangs- und Zieldomäne in der CTM sowie für mentale Räume fungieren zu können:

The phenomena generally dealt with in mental space theory, and the analyses proposed, are readily accommodated in CG. This is actually quite evident, since any kind of conception counts as a domain, and no restrictions are imposed on how the domains of a matrix are related to one another. Thus any mental space configuration including both the spaces and the connections linking them - can simply be incorporated as part of a matrix.

(Langacker 2008: 51)

\footnotetext{
${ }^{9}$ Das Ziel der CTM liegt in der Suche nach grundlegenden universalen konzeptuellen Strukturen, die unser Denken und unsere Sprache in verschiedenen Bereichen strukturieren. Die konzeptuelle Metapher wird hier als eine grundlegende konzeptuelle Kategorie, "a mode of thought" (Lakoff 1993: 210) verstanden, die unser Denken organisiert und sich in der Sprache in einer Anzahl der Sprachausdrücke manifestiert. Die Theorie der mentalen Räume und die Blending-Theorie sind dagegen auf den Diskurs ausgerichtet.

10 Langacker (2008: 51) erklärt den subtilen Unterschied zwischen der Domäne und dem mentalen Raum auf folgende Weise: "As a way of referring to conceptual content, it [a domain, A.S.] tends to be used for established conceptions in relation to lexical meanings. By contrast, mental space emphasizes conceptual discontinuities, the partitioning of conceptual structure into semiautonomous regions. It tends to be employed for the products of imaginative operations and the structures created dynamically in discourse. These are only tendencies, however, and both terms are vague enough for general application."
} 
Somit kann man die Metapher im Rahmen der KG als eine Domänenmatrix darstellen, deren Einzigartigkeit darin besteht, dass manche ihrer Domänen durch Ähnlichkeitsbeziehungen verbunden werden. $\mathrm{Zu}$ der Konzeptualisierung der Zange in einer Äußerung mit der ad-hocMetapher

(3) Lisa stellte den Motor ab und hoffte, er möge wieder anspringen. Sie gab ihren Ausweis in die Zange [Hervorhebung: A.S.] der beiden Finger, die durch den schmalen Schlitz lugten. Wohin reisen Sie?

(Jentzsch, Kerstin (1999): Seit die Götter ratlos sind, zit. nach DWDS, s. v.in\&\&@die\&\&Zange\&\&nehmen)

könnten dementsprechend folgende Domänen beitragen: die Domäne der Verkehrs- bzw. Grenzkontrolle, die Domäne von Dokumenten, die Domäne der Polizisten/der Zollbeamten sowie zwei durch Ähnlichkeitsrelationen verbundenen Domänen der prototypischen Zange und der menschlichen Hand als eines Greiforganes, der einen Pinzettengriff ermöglicht. Jede der zwei zuletzt genannten Domänen ist komplex und aus anderen hierarchisch untergeordneten (Sub-)Domänen zusammengesetzt. Zur Vermeidung von terminologischen Schwierigkeiten werden die durch Ähnlichkeitsbeziehungen gebundenen Domänen im Folgenden als Ausgangsbereich (die komplexe Domäne der Zange) und als Zielbereich (die Domäne der Hand) bezeichnet. Ausschlaggebend für die Konzeptualisierung sind demzufolge nur ausgewählte Domänen des Ausgangsbereiches: die Basisdomäne des Raumes, die Domäne der Form (zwei Finger wie zwei Greifbacken einer Zange), die Domäne, die das Wissen aktiviert, wie eine Zange bedient wird (ein Werkzeug in einer Hand eines Menschen), die Domäne des präzisen Festhaltens mit Hilfe von zwei Fingern (dem Daumen und dem Zeigefinger). Selbstverständlich bildet dieses Wissen nur einen Teil des konzeptuellen Inhaltes von dem viel komplexeren Konzept Hand, allerdings ist es dieser Teil, der zum Verstehen dieser konkreten Äußerung und zur Bildung der Konzeptualisierung notwendig ist. Die Relation zwischen den durch Ähnlichkeitsstrukturen verbundenen Ausgangs- und Zielbereichen sowie anderen zur Konzeptualisierung beitragenden Domänen veranschaulicht folgendes Schema:

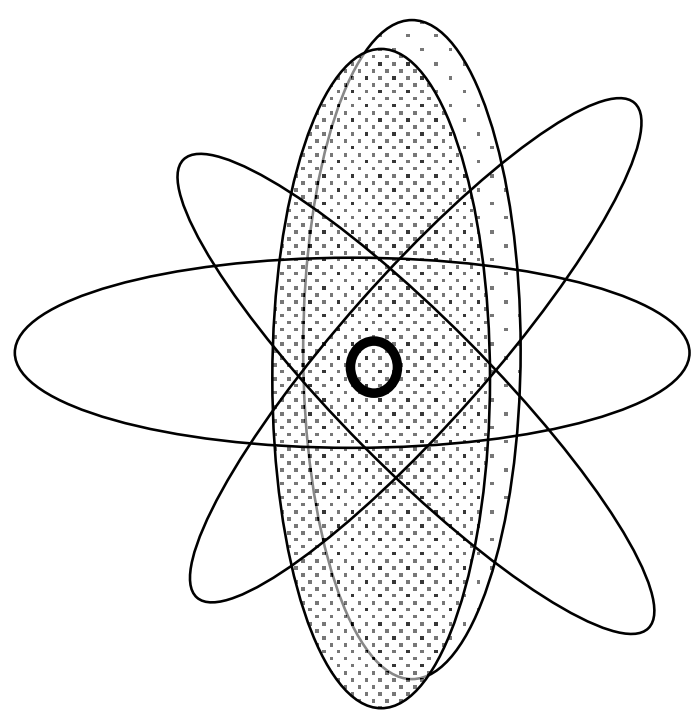

Abb. 3: Konzeptualisierung einer ad-hoc Metapher (schattiert sind die durch Ähnlichkeitsbeziehungen gebundenen Domänen des Ausgangs- und Zielbereiches dargestellt) 


\section{Die Bedeutungskonstituierung metaphorisch motivierter Idiome}

Der oben besprochene Gebrauchsbeleg fokussierte auf der Konzeptualisierung einer ad-hocMetapher, einem vereinzelten idiosynkratischen Sprachgebrauch. In der Sprache gibt es aber auch viele lexikalisierte Spracheinheiten, darunter Idiome, denen eine Metapher zu Grunde liegt.

Im Fokus unseres Interesses befindet sich im Folgenden ein metaphorisch motiviertes Idiom: jmdn. in die Zange nehmen, das in Anlehnung an weitere Gebrauchsbelege aus dem DWDSKorpus untersucht wird. In den Mittelpunkt des Interesses wird dabei die Relation zwischen den metaphorisch gebundenen Domänen des Ausgangs- und Zielbereiches gerückt: Es wird der Frage nachgegangen, ob bzw. inwieweit und welche Domänen bei der Konstituierung der Idiom-Bedeutung im authentischen Sprachgebrauch zu Geltung kommen. Da die Analyse aller Gebrauchsbelege aus dem DWDS-Kernkorpus den Rahmen dieses Beitrags sprengen würde, gehen wir auf ausgewählte Usus-Beispiele ein. ${ }^{11}$

\section{1 'jmdn. (den Feind) von zwei Seiten umgeben, von seinen Nachschub- verbindungen abschneiden und einkesseln'}

In insgesamt sechs Gebrauchsbelegen wird mit Hilfe des Idioms: jmdn in die Zange nehmen bildlich eine bewährte Kriegsstrategie dargestellt, in der der Feind von seinen Nachschubverbindungen abgeschnitten, eingekesselt und, wenn möglich, vernichtet wird:

(4) Das mag grotesk klingen; aber, um diese Front von über 2000 Kilometer überhaupt zu besetzen, brauchte man mindestens 300 Divisionen, und die haben wir nie gehabt. Wenn wir gewartet hätten, bis wir vielleicht gleichzeitig durch die Invasion und durch einen russischen Angriff in die Zange genommen worden wären, so wären wir mit Sicherheit verloren gewesen.

(s. n. (1946/1999): Der Nürnberger Prozeß, zit. nach DWDS, s. vin\&\&@die\&\&Zange\&\&nehmen)

Einfluss auf die Konzeptualisierung haben hier z. B. die Domänen der Kriegsführung, der Logistik, des zweiten Weltkrieges, des Todes. Besonders deutlich kommt hier aber auch das mentale Vorstellungsbild der sich schließenden Zange zum Vorschein, die das Patiens zuerst isoliert, dann gefangen nimmt, der Fluchtmöglichkeiten beraubt und am Ende zerdrückt. Fokussiert werden die perzeptuellen (2) sowie funktionalen Domänen (4, 5, 6, 7) des Konzeptes Zange in der wörtlichen Lesart, sowie die Domäne der Folter und des Schmerzens (11), den die Fixierung eines lebendigen Organismus mit einer Zange auslösen müsste. Somit ist die Konzeptualisierung der Zange als eines aus zwei Greifbacken bestehenden Gegenstandes zur präzisen Fixierung eines Objektes, des durch das Hebelprinzip verstärkten Druckausübens

\footnotetext{
11 Einer detaillierten Untersuchung wurden 19 zugängliche Kernkorpusbelege für den Phraseologismus jmdn. in die Zange nehmen unterzogen (Suchanfrage: "in\&\&@die\&\&Zange\&\&nehmen" [12.01.14]). Beachtenswert und lexikographisch relevant sind vor allem die im Punkt 4.1, 4.3, 4.4 besprochenen Bedeutungen, da sie mit mehreren Gebrauchsbelegen vertreten sind und Verwendungsprofile bilden: Somit wird das Idiom insgesamt 6 Mal in der kontextuellen Einbettung einer Kriegsstrategie gebraucht, in 4 Gebrauchsbelegen bezieht es sich auf den durch eine Person in höheren Position ausgeübten Druck, ebenfalls in 4 Belegen wird das Idiom in Bezug auf ein Verhör, eine intensive Befragung eines Betroffenen zur Klärung einer unangenehmen Situation verwendet.
} 
von beiden Seiten ${ }^{12}$, der großen Kraft, des Schmerzes, der vom Patiens gespürten Existenzbedrohung ausschlaggebend.

\begin{tabular}{|l|l|}
\hline Ausgangsbereich & Zielbereich \\
\hline $\begin{array}{l}\text { Domäne 2: } \\
\text { zwei Bearbeitungsflächen, mit } \\
\text { denen ein Gegenstand von zwei } \\
\text { Seiten zugleich fixiert und bear- } \\
\text { beitet werden kann }\end{array}$ & $\begin{array}{l}\text { Zwei Seiten, aus denen zugleich der Angriff erfolgt. } \\
\text { Die Bearbeitungsflächen bilden die feindlichen Trup- } \\
\text { pen, dem zu bearbeitenden Gegenstand entsprechen } \\
\text { die eingekesselten Soldaten. }\end{array}$ \\
\hline $\begin{array}{l}\text { Domäne 3: } \\
\text { Ein Werkzeug, dass von einer } \\
\text { Person intentional bedient wird }\end{array}$ & $\begin{array}{l}\text { Eine Befehlsgewalt (ein Kommando), die die Operati- } \\
\text { on überblickt und anführt. }\end{array}$ \\
\hline $\begin{array}{l}\text { Domäne 4: } \\
\text { Hebelprinzip: durch den Druck zwei Schenkel vermehren } \\
\text { sich die wirkenden Kräfte }\end{array}$ & $\begin{array}{l}\text { Durch die doppelseitige Invasion militärischer Einhei- } \\
\text { ten ist der Angriff verstärkt, kräfteraubend. }\end{array}$ \\
\hline $\begin{array}{l}\text { Domäne 5: } \\
\text { Festhalten von Objekten }\end{array}$ & $\begin{array}{l}\text { Die eingekesselten Soldaten können dem Angriff nicht } \\
\text { ausweichen, sie können nicht entfliehen. }\end{array}$ \\
\hline $\begin{array}{l}\text { Domäne 6: } \\
\text { Abschneiden von Gegenständen }\end{array}$ & $\begin{array}{l}\text { Am Ende der Operation wird das Patiens isoliert, von } \\
\text { seinen Nachschubverbindungen abgeschnitten. } \\
\text { Die feindlichen Truppen umfassen ihn wie die Greif- } \\
\text { backen einer Eckzange: Wenn sich die Zange schließt, } \\
\text { ist die Situation hoffnungslos. }\end{array}$ \\
\hline $\begin{array}{l}\text { Durch die Überzahl kann der Feind die Frontlinie be- } \\
\text { liebig gestalten. } \\
\text { Durch den psychischen Druck, die Ausweglosigkeit } \\
\text { der Situation werden die Soldaten zur Waffen- } \\
\text { streckung veranlasst. }\end{array}$ \\
\hline $\begin{array}{l}\text { Diegen von Objekten } \\
\text { Quetschen Truppen werden durch den Zwang auf einen im- } \\
\text { richtung }\end{array}$ & $\begin{array}{l}\text { Die Existenz der Soldaten ist bedroht, sie sind in Le- } \\
\text { bensgefahr. Das Geschehen zerrt an körperlichen und } \\
\text { psychischen Kräften des Patiens, kann zu irrationalen } \\
\text { Schritten führen. }\end{array}$ \\
\hline
\end{tabular}

Tab. 1: Metaphorische Mappings zwischen dem Ausgangs- und Zielbereich für die aktuelle Bedeutung 'jmdn. (den Feind) von seinen Nachschubverbindungen abschneiden und einkesseln'

Wie aus der Tabelle 1 ersichtlich ist, werden in diesem Gebrauchsbeleg zahlreiche Domänen des Ausgangsbereiches in das Zielbereich übertragen. Bemerkenswert ist die zeitliche und räumliche Komprimierung des Geschehens: Während die Fixierung eines Objektes mit einer Zange von kurzer, von einem Individuum leicht überschaubarer Dauer (und deswegen erfahrungsnah) ist, kann sich eine militärische Operation über Monate und weite Gebiete erstrecken.

\footnotetext{
12 Der durch die bildliche Komponente motivierte Aspekt des Druckausübens von zwei Seiten spielt in den meisten Belegen eine große Rolle. In zahlreichen neueren Belegen des Zeit\&Zeit-online-Korpuses wird er explizit zum Ausdruck gebracht, z. B.: "Die bäuerlichen Biogasproduzenten - sie stellen bislang noch die Mehrzahl der Anlagenbetreiber - sehen sich von zwei Seiten in die Zange genommen [Hervorhebung: A.S.]. Zum einen vom Weltmarkt, zum anderen von den großen Energieversorgern [...]" (Die Zeit 20.05.08, zit. nach DWDS, s. v. Zange).
} 


\section{2 'auf jmdn. (eine Partei) von zwei Seiten Druck ausüben und sie in eine ausweglose Lage bringen'}

Viele Gemeinsamkeiten mit dem besprochenen vierten Gebrauchsbeleg scheint auf den ersten Blick der fünfte Korpusbeleg zu haben: Der Druck von zwei Seiten auf eine anarchistische Partei CNT verursacht, dass auf wichtige Voraussetzungen bzw. leitende Prinzipien Verzicht geleistet wird:

(5) Die Führung der CNT-FAI war der Situation, in der sie sich im Herbst 1936 fand, in keiner Weise gewachsen. Von der faschistischen Offensive einerseits und von der Konterrevolution im eigenen Lager andererseits in die Zange genommen [Hervorhebung: A.S.], konnte sie an den schlichten, hergebrachten Prinzipien der anarchistischen Lehre nicht mehr ohne Abstriche festhalten. Sie ist vor den Realitäten Schritt für Schritt zurückgewichen.

(Enzensberger, Hans Magnus (1972): Der kurze Sommer der Anarchie, zit. nach DWDS, s. v.in\&\&@die\&\&Zange\&\&nehmen)

Der Einfluss der Domänen des Ausgangsbereiches auf den Zielbereich ist in diesem Gebrauchsbeleg dennoch nuancierter und abstrakter als in der vierten Textstelle: Während es in dem obigen Beispiel eine feindliche Kraft gibt bzw. die feindlichen Kräfte ein gemeinsames Ziel verfolgen, sind hier die druckausübenden Kräfte keinesfalls gleich. Die schwierige Lage der Führung einer anarchistischen Partei wird durch Faschisten einerseits und Konterrevolution in eigenen Reihen andererseits hervorgerufen - infolgedessen ist die Intentionalität der Handlung gestört. Daraus lässt sich schlussfolgern, dass es keine Person gibt, die für die schwierige Situation zuständig ist (d.h. die Zange in der Hand hält). Die zu projizierende Zange hat infolgedessen zwei Greifbacken, die dennoch durch keinen Drehbolzen verbunden und nicht von einer Person hantiert werden. Das mentale Vorstellungsbild entbehrt somit wichtiger Eigenschaften des Gegenstandes, die in den perzeptuellen Domänen repräsentiert werden. Profiliert werden dafür die funktionalen Domänen:

\begin{tabular}{|l|l|}
\hline Ausgangsbereich & Zielbereich \\
\hline $\begin{array}{l}\text { Domäne 4: } \\
\text { Hebelprinzip }\end{array}$ & Der Druck von beiden Seiten ist sehr stark. \\
\hline $\begin{array}{l}\text { Domäne 5: } \\
\text { Festhalten von Objekten }\end{array}$ & $\begin{array}{l}\text { Ausweglosigkeit der Situation aus der Sicht der } \\
\text { Parteiführung, die keine Schritte unternehmen } \\
\text { kann. }\end{array}$ \\
\hline $\begin{array}{l}\text { Domäne 7: } \\
\text { Biegen von Objekten }\end{array}$ & $\begin{array}{l}\text { Die Parteiführung muss sich in gegebene Verhält- } \\
\text { nisse einordnen und vor dem psychologischen } \\
\text { Druck zurückweichen, d. h. wichtige Prinzipien } \\
\text { aufgeben. }\end{array}$ \\
\hline $\begin{array}{l}\text { Domäne 9: } \\
\text { Quetschen von Objekten }\end{array}$ & $\begin{array}{l}\text { Die ideologische Freiheit der Parteiführung ist ein- } \\
\text { geschränkt. Sie darf ihre radikalen Ideen nicht mehr } \\
\text { verbreiten - im Gegensatz - unter dem psychologi- } \\
\text { schen Druck muss sie sogar darauf allmählich ver- } \\
\text { zichten. }\end{array}$ \\
\hline $\begin{array}{l}\text { Domäne 12: } \\
\text { Werkzeug zur Folter und } \\
\text { Hinrichtung }\end{array}$ & $\begin{array}{l}\text { Die Situation ist für die Betroffenen extrem schwie- } \\
\text { rig, der Verzicht auf revolutionäre Ziele wahr- } \\
\text { scheinlich sehr schmerzhaft. }\end{array}$ \\
\hline
\end{tabular}

Tab. 2: Metaphorische Mappings zwischen dem Ausgangs- und Zielbereich für die aktuelle Bedeutung 'auf jmdn. (eine Partei) von zwei Seiten Druck ausüben und sie in eine ausweglose Lage bringen' 


\section{3 'jd. (eine machtausübende Person) zwingt jmdn. zu einer unerwünschten Entscheidung'}

Mit dem weiteren Gebrauchsbeleg ist die Bedeutung vertreten, die man mit den Worten: 'von einer machtausübenden Person zu einer unerwünschten Entscheidung gezwungen werden' paraphrasieren könnte. Das Geschehen wird hier nicht durch abstrakte Kräfte, sondern durch einen einflussreichen Menschen bestimmt, der über Mittel und Methoden verfügt, mit denen andere fügsam gemacht werden:

(6) Und die Kunde ging, der Fraktionsvorsitzende habe mich in die Zange genommen [Hervorhebung: A.S.] und zum Rücktritt veranlaßt. Begründung: Ich sei erpreßbar.

(Brandt, Willy (1989/97): Erinnerungen, zit. nach DWDS, s. v.in\&\&@die\&\&Zange\&\&nehmen)

Einfluss auf die Konzeptualisierung haben hier die Domänen der parlamentarischen Struktur, der Hierarchie und der Karriere in der Politik, des politischen Erfolgs und Misserfolgs. Fokussiert man die Beziehungen zwischen den metaphorisch gebundenen Domänen, dann sind auch in diesem Gebrauchsbeleg die funktionalen Wissensbereichen von Relevanz:

\begin{tabular}{|l|l|}
\hline Ausgangsbereich & Zielbereich \\
\hline $\begin{array}{l}\text { Domäne 3: } \\
\text { intentional bedient wird }\end{array}$ & $\begin{array}{l}\text { Eine Person, die durch ihre höhere politische und } \\
\text { gesellschaftliche Stellung befugt ist, über Menschen } \\
\text { und Verhältnisse zu bestimmen. }\end{array}$ \\
\hline $\begin{array}{l}\text { Domäne 4: } \\
\text { Hebelprinzip }\end{array}$ & Der ausgeübte Druck ist sehr stark. \\
\hline $\begin{array}{l}\text { Domäne 5: } \\
\text { Festhalten von Objekten }\end{array}$ & $\begin{array}{l}\text { Der Betroffene muss sich der unangenehmen Ausei- } \\
\text { nandersetzung mit dem Fraktionsvorsitzenden stel- } \\
\text { len. }\end{array}$ \\
\hline $\begin{array}{l}\text { Domäne 7: } \\
\text { Biegen von Objekten }\end{array}$ & $\begin{array}{l}\text { An dem Betroffenen wird eine Entscheidung er- } \\
\text { zwungen, die für ihn ungünstig ist. Der Betroffene } \\
\text { muss sich in gegebene Verhältnisse einordnen, er } \\
\text { muss sich fügen. }\end{array}$ \\
\hline $\begin{array}{l}\text { Domäne 9: } \\
\text { Quetschen von Objekten }\end{array}$ & $\begin{array}{l}\text { Der Agens "bearbeitet" seinen Mitarbeiter so lange, } \\
\text { verstärkt den Druck dermaßen, dass der Betroffene } \\
\text { endlich nachgeben muss, zum Gehorsam gezwungen } \\
\text { wird. }\end{array}$ \\
\hline $\begin{array}{l}\text { Domäne 12: } \\
\text { Werkzeug zur Folter und Hinrich- } \\
\text { tung }\end{array}$ & $\begin{array}{l}\text { Der Betroffene wird dem psychischen Druck ausge- } \\
\text { setzt. }\end{array}$ \\
\hline
\end{tabular}

Tab. 3: Metaphorische Mappings zwischen dem Ausgangs- und Zielbereich für die aktuelle Bedeutung 'jd. (eine machtausübende Person) zwingt jmdn. zu einer unerwünschten Entscheidung'

\section{4 'jmdn. mit gezielt gestellten Fragen zu einer unangenehmen Bekenntnis zwingen'}

Stellvertretend für vier weitere Gebrauchsbelege, in denen die Bedeutungsnuance: 'jmdn. mit gezielt gestellten Fragen zu einer unangenehmen Bekenntnis zwingen' wird folgendes UsusBeispiel analysiert:

(7) Zu später Stunde, im ARD-Kulturmagazin "Titel, Thesen, Temperamente", nahm Buchheim, 69, den "Landbriefträger" Nebel, 45, schwer in die Zange [Hervorhebung A.S.]. Waren Sie in Ihrem Leben überhaupt schon mal in einer bedeutenden Kunstsammlung? inquirierte 
Buchheim. Und als die Antwort vage blieb, schoß der ehemalige Leutnant zur See den braven Land-Mann unerbittlich in den Grund: "Man kann mit einem Brunnenfrosch nicht vom Ozean reden."

(Der Spiegel 18.01.88, zit. nach DWDS, s.

v.in\&\&@die\&\&Zange\&\&nehmen)

Die Agensrolle übernimmt hier ein Mann (Schriftsteller, Maler, Verleger), der seinen Gesprächspartner vor laufenden Kameras der Ignoranz überführt. Von Relevanz für die Konzeptualisierung sind hier außer der Domänen der Diskussion, der Liveübertragung auch die funktionalen Domänen, die durch das Konzept Zange evoziert werden: die Domänen des Festhaltens, des Quetschens und der Folter:

\begin{tabular}{|l|l|}
\hline Ausgangsbereich & Zielbereich \\
\hline $\begin{array}{l}\text { Domäne 3: } \\
\text { Ein Werkzeug, dass von einer Person } \\
\text { intentional bedient wird }\end{array}$ & $\begin{array}{l}\text { In einem Fernsehgespräch kontrolliert eine Person } \\
\text { die Situation. }\end{array}$ \\
\hline $\begin{array}{l}\text { Domäne 4: } \\
\text { Hebelprinzip }\end{array}$ & Die Intensivierung des ausgeübten Drucks. \\
\hline $\begin{array}{l}\text { Domäne 5: } \\
\text { Festhalten von Objekten }\end{array}$ & $\begin{array}{l}\text { Der Agens ist sich dessen bewusst, dass das Patiens } \\
\text { in dieser Situation (Fernsehstudio, Live-Sendung) } \\
\text { ihm nicht entweichen kann. Er nutzt die Umstände, } \\
\text { um ihn mit Fragen in eine peinliche Lage zu brin- } \\
\text { gen. }\end{array}$ \\
\hline $\begin{array}{l}\text { Domäne 8: } \\
\text { Quetschen }\end{array}$ & $\begin{array}{l}\text { Der Agens kann die Druckkraft regulieren, er kon- } \\
\text { trolliert die Situation. Durch gezielte Entschei- } \\
\text { dungsfragen wird der Druck verstärkt. }\end{array}$ \\
\hline $\begin{array}{l}\text { Domäne 12: } \\
\text { Folter und Hinrichtung }\end{array}$ & $\begin{array}{l}\text { So wie bei Folter der Betroffene oder seine Körper- } \\
\text { teile dem physischen Druck (zusammendrücken, } \\
\text { quetschen) ausgesetzt sind, so ist wird hier auf den } \\
\text { Patiens hier der psychische Druck ausgeübt. Die } \\
\text { Lage des Patiens ist höchst unangenehm. }\end{array}$ \\
\hline
\end{tabular}

Tab. 4: Metaphorische Mappings zwischen dem Ausgangs- und Zielbereich für die aktuelle Bedeutung 'jmdn. mit gezielt gestellten Fragen zu einer unangenehmen Bekenntnis zwingen'

\section{5 'in einem Konkurrenzkampf den Konkurrenten in eine schwierige Lage bringen'}

Im letzten Gebrauchsbeleg ist die Bedeutung des Idioms relativ vage und die metaphorische Verbindung zwischen dem Ausgangs- und dem Zielbereich am schwersten nachvollziehbar:

(8) 1967 wurde Ariel auf den deutschen Markt geworfen und als Vorwaschmittel "positioniert": "Zum Vorwaschen gibt's doch was Besonderes. Denn Ariel kann reinweichen". Damit hatte Procter\&Gamble den Marktführer Persil in die Zange genommen.

(Hars, Wolfgang (1999/2001): Nichts ist unmöglich! Lexikon der Werbesprüche, zit.nach DWDS, s. v. in\&\&@die\&\&Zange\&\&nehmen)

Einfluss auf die Konzeptualisierung könnte auch hier vor allem der funktionalen Domäne des Quetschens zugeschrieben werden, der Spielraum der möglichen Interpretationen ist dennoch größer als in den bisher besprochenen Belegen. 


\begin{tabular}{|l|l|}
\hline Ausgangsbereich & Zielbereich \\
\hline $\begin{array}{l}\text { Domäne 3: } \\
\text { Ein Werkzeug, dass von einer Person } \\
\text { intentional bedient wird }\end{array}$ & $\begin{array}{l}\text { Ein Konzern, der im Konkurrenzkampf einen ande- } \\
\text { ren Konzern intentional behindert, um Machtposition } \\
\text { und größere Gewinne zu erzielen. }\end{array}$ \\
\hline Domäne 9: & Im Wettbewerb verschiedener Anbieter um Kunden \\
Quetschen von Objekten & gibt die Einführung einer Innovation auf den Markt \\
& dem einführenden Konzern einen Vorsprung, ver- \\
& leiht ihm die Position eines Vorreiters. Somit wird \\
& zeitweise der Einfluss der Konkurrenz auf den Markt \\
& und die Nachfrage nach ihren Produkten einge- \\
& schänkt, was auch ihr Einkommen und ihre Ent- \\
& wicklungschancen beeinflusst. \\
\hline
\end{tabular}

Tab. 5: Metaphorische Mappings zwischen dem Ausgangs- und Zielbereich für die aktuelle Bedeutung 'in einem Konkurrenzkampf den Konkurrenten in eine schwierige Lage bringen'

\subsection{Zusammenfassung: Die Bedeutungskonstituierung metaphorisch motivierter Idiome}

Wie aus der Analyse ersichtlich ist, variieren die Konzeptualisierungen der ZANGE in den Gebrauchsbelegen stark in der Anzahl der Verbindungen zwischen den metaphorisch gebundenen Domänen des Ausgangs- und des Zielbereiches, in der inneren Kohärenz des mentalen Bildes, in der Vagheit der Bedeutung. Demgemäß werden beispielshalber im Gebrauchsbeleg (4) acht Domänen des Ausgangsbereiches durch metaphorische Projizierung in den Zielbereich übertragen, der Anteil der perzeptuellen Domänen bei der Konstituierung der Konzeptualisierung ist sehr deutlich, die metaphorische Übertragung zwischen dem Ausgangs- und Zielbereich schlüssig, und das evozierte mentale Bild trägt zur präzisen Beschreibung des Geschehens bei: Die Greifbacken der Zange sind von feindlichen militärischen Einheiten gebildet, die Schenkel der Zange hat ein Kommando im Griff, das wahrscheinlich aus gewisser Entfernung die ganze Operation regiert, das anvisierte Ziel der Operation (das Umringen, Abschneiden der Fluchtwege, Zerstören der feindlichen Streitkräfte) liegt hier auf der Hand. Mit größter Wahrscheinlichkeit könnte angenommen werden, dass der Satz auch von einem Sprachteilhaber verstanden würde, der das Idiom nicht kennt. Auf dem anderen Pol steht der Gebrauchsbeleg (8), bei dem die metaphorische Projizierung zwischen dem Ausgangs- und Zielbereich auf lediglich zwei Domänen eingeschränkt ist, was die Interpretation anhand der Metapher schwer macht: Zur Rezeption des Idioms in diesem Beleg ist das Heranziehen einer zusätzlichen Domäne - der Domäne der lexikalisierten Bedeutung - notwendig.

Im Gegensatz zu den ad-hoc Metaphern, deren Verstehen ausschließlich durch den Rückgriff auf den Kontext, auf das von dem Sprecher und dem Rezipienten geteilte Wissen und die Herstellung neuartiger Beziehungen zwischen dem Ausgangs- und Zielbereich möglich ist, verfügen die metaphorisch motivierten Idiome also ebenfalls über eine lexikalisierte, auf Grund der Konvention festgelegte Bedeutung. Diese Bedeutung erschließt der Sprecher im Spracherwerbsprozess größtenteils aus den Sprachgebrauchsereignissen auf der Grundlage der Schematisierung: Dementsprechend beziehen sich Schemata auf eine Abstraktion und Verstärkung der Regularitäten aus den Gebrauchserfahrungen (Konkretisierungen), was Taylor (2007: 148) in Form eines Dreiecks darstellt: 


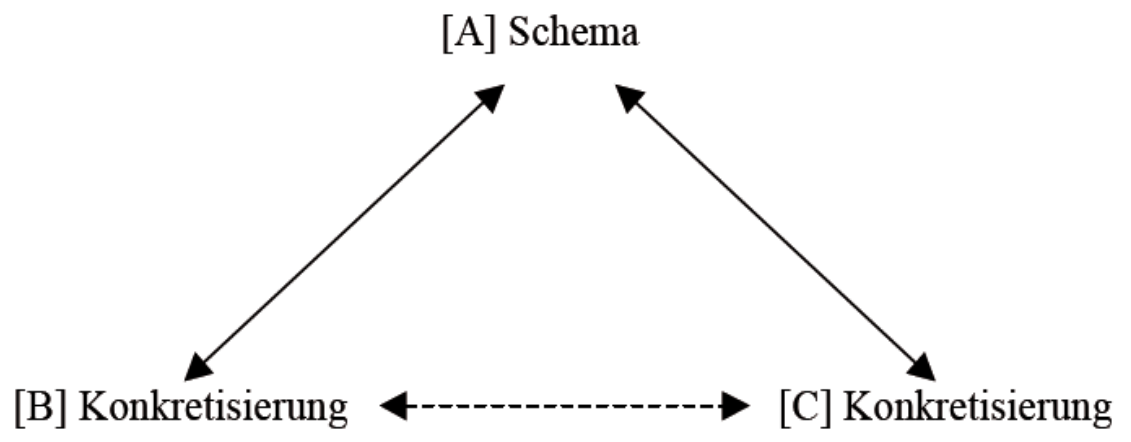

Abb. 4: Schema und Konkretisierungen nach Taylor (2007: 148)

Die Existenz des übergeordneten Schemas erklärt die nachträgliche Interpretierbarkeit der Idiome sowie die problematische Beziehung zwischen der Motiviertheit und der Arbitrarität der idiomatischen Spracheinheiten: Die Beziehung zwischen dem Ausdruck und der Bedeutung ist in den metaphorisch motivierten Idiomen für den Sprecher nachträglich leicht erkennbar und einleuchtend, lässt sich aber nicht immer im Voraus aus der Form prognostizieren, was Dobrovol'skij (2001: 90) folgendermaßen ausdrückt: "Die Sprache ist folglich arbiträr und nichtarbiträr zugleich: arbiträr im Sinne der Imprediktabilität und nichtarbiträr im Sinne der nachträglichen Interpretierbarkeit relevanter Phänomene."

Bei der Konzeptualisierung eines Idioms wird infolgedessen außer den metaphorisch gebundenen Ausgangs- und Zieldomänen sowie den kontextbedingten Wissensbereichen auch die Domäne aktiviert, in der die aus den bisherigen Gebrauchserfahrungen abstrahierte lexikalisierte Bedeutung aufbewahrt wird, womit der Spielraum für die möglichen Interpretationen einer Metapher eingeschränkt wird.

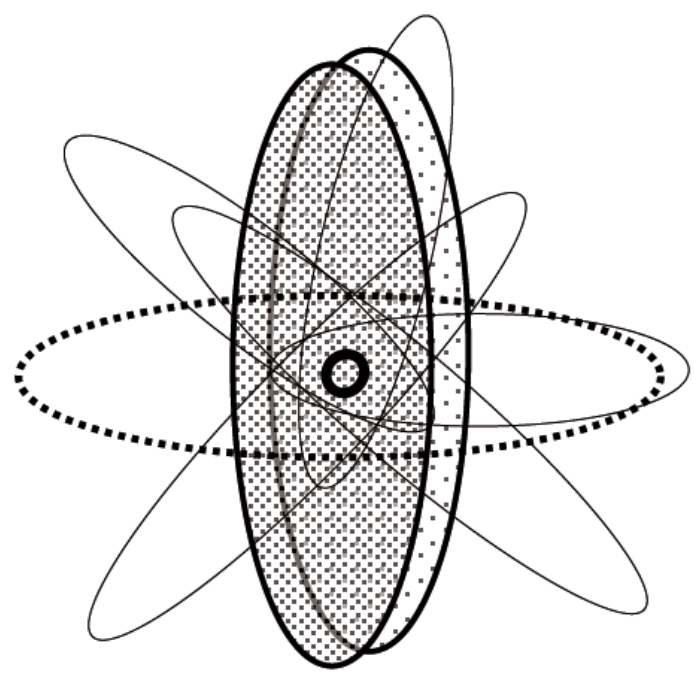

Abb. 5: Konzeptualisierung eines metaphorisch motivierten Idioms (schattiert sind die durch Ähnlichkeitsbeziehungen gebundenen Domänen dargestellt - in Form einer gepunktet umrandeten Ellipse die Domäne der lexikalisierten Bedeutung)

Der Anteil der Domäne der lexikalisierten Bedeutung mag bei der Konzeptualisierung unterschiedlich sein und hängt zum Teil auch damit zusammen, wie einleuchtend für einen Sprachteilhaber die Metapher ist - die intersubjektiv fassbaren Kriterien lassen sich hier wahrscheinlich nicht aufstellen. Der Übergang zwischen einer motivierten Metapher und einer metapho- 
risch motivierten Mehrworteinheit mit lexikalisierter Bedeutung sind deswegen individuell und verschwommen - hier liegen eher Abstufungen als Dichotomien vor.

\section{$5 \quad$ Resümee}

Den Konzeptualisierungen werden im Rahmen der Kognitiven Grammatik dieselben Eigenschaften wie dem Weltverstehen zugeschrieben. Sie sind dynamisch, aktiv, haben einen individuellen, konstruktivistischen Charakter und verschwommene Grenzen (vgl. Langacker 2008: 35). Diese Eigenschaften weisen auch die Konzeptualisierungen der Zange in allen besprochenen Gebrauchsbelegen - sowohl in der wörtlichen als auch in der lexikalisierten, phraseologischen Bedeutung - auf. In Bezug auf das Idiom jmdn. in die Zange nehmen lässt sich dabei bemerken, dass die Domänen der Hauptkonstituente Zange in der wörtlichen Lesart zwar in unterschiedlichem Maße, aber dennoch in jedem Gebrauchsbeleg von Relevanz für die Konzeptualisierung sind. Somit können die metaphorisch motivierten Idiome als sekundäre Sprachzeichen betrachtet werden, die aufgrund der doppelten Repräsentation auf dem Inhaltsplan eine komplexere semantische Struktur als primäre Sprachzeichen aufweisen: Die Konstituierung der phraseologischen Bedeutung von einem Idiom erfolgt (mehr oder weniger) auf der Folie seiner wörtlichen Bedeutung, was das folgende Schema veranschaulicht:

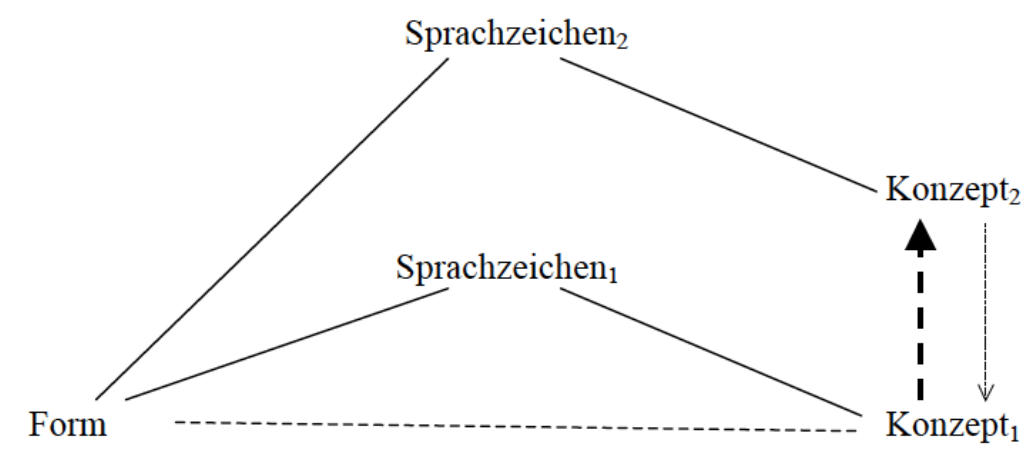

Abb. 6: Metaphorische Idiome als sekundäre Sprachzeichen

Primär ist die Verbindung vom Konzept ${ }_{1}$ zum Konzept ${ }_{2}$, die aufgrund der metaphorischen Beziehungen zwischen dem Ausgangs- und Zielbereich entsteht. Der häufige Gebrauch eines Idioms kann dennoch ebenfalls zur Bedeutungserweiterung der Hauptkonstituente führen. Als Beispiel könnte man an dieser Stelle die Hauptkonstituente der Idiome jmdn. in die Patsche reiten; in der Patsche sitzen heranführen. Die idiomatische Bedeutung der Patsche hat die wörtliche Bedeutung verdrängt, was sich in der lexikographischen Auffassung des Lexems widerspiegelt. ${ }^{13}$ Hinsichtlich der metaphorisch motivierten Idiome ist folglich Chlebda (2001: 431) zuzustimmen, der darauf verweist, dass sich die Bedeutung der Idiome zwischen den beiden Polen: dem Pol der wörtlichen und dem Pol der phraseologischen Bedeutung bewegt:

Beide Bedeutungen koexistieren sowohl im Geiste des Senders als auch im Geiste des Empfängers einer Aussage. Phrasematische Beobachtungen führen vor Augen, dass die "semantische Zweigleisigkeit" die reale Wirklichkeit unserer Alltagskommunikation ausmacht. Dieser eigentümliche "Zustand der semantischen Schwankung" zwischen dem Pol der Wörtlichkeit

13 vgl. Duden (2007) s. v. Pạt|sche, die; -, -n [4: eigtl. = Matsch, aufgeweichte Straße] (ugs.): 1. Hand, 2. Feuerpatsche, 3. Patsch $\uparrow, 4$. <Pl. selten> unangenehme, schwierige Lage, Bedrängnis: jmdm. aus der P. helfen; in der P. sitzen. 
und der Übertragenheit wird in der Phrasematik als natürlich und objektiv feststellbar betrachtet. $^{14}$

(Chlebda 2001: 431)

\section{Literatur}

Berlin-Brandenburgische Akademie der Wissenschaften (ed.) (2010-): Digitales Wörterbuch der deutschen Sprache. www.dwds.de [14.01.14].

Burger, Harald (2010): Phraseologie. Eine Einführung am Beispiel des Deutschen. 4. Auflage. Berlin: Erich Schmidt.

Chlebda, Wojciech (2001): "Frazematyka". In: Bartmiński, Jerzy (ed.): Współczesny język polski. Lublin, Wydawnictwo UMCS: 335-342.

Dobrovol'skij, Dmitrij (2001): "Zur Motivation in der Idiomatik". In: Häcki Buhofer, Annelies/Burger, Harald/Gautier, Laurent (ed.): Phraseologiae Amor. Aspekte europäischer Phraseologie. Festschrift für Gertrud Gréciano zum 60. Geburtstag. Hohengehren, Baltmannsweiler: 89-98.

Duden (2007): Deutsches Universalwörterbuch. www.duden.de/woerterbuch [13.01.14].

Duden (2011): Redewendungen. Wörterbuch der deutschen Idiomatik. 3. Auflage. Mannheim usw.: Duden.

Fauconnier, Gilles (1985): Mental Spaces: Aspects of Meaning Construction in Natural Language. London: Bradford.

Fauconnier, Gilles (1997): Mappings in Thought and Language. Cambridge: Cambridge University Press.

Lakoff, George (1993): "The contemporary theory of metaphor". In: Ortony, Andrew (ed.): Metaphor and Thought. 2. Auflage. Cambridge: Cambridge University Press: 202-251.

Lakoff, George/Johnson, Mark (1980): Metaphors we live by. Chicago: University of Chicago Press.

Langacker, Ronald R. (2008): Cognitive Grammar. A Basic Introduction. Oxford: Oxford University Press.

Langacker, Ronald R. (2009): Gramatyka kognitywna. Wprowadzenie. (aus dem Engl. übersetzt von E. Tabakowska, M. Buchta, H. Kardela u. a.). Kraków: Universitas.

Menzel, Wolfgang (1834): Geschichte der Deutschen bis auf die neuesten Tage. Stuttgart/Tübingen: Verlag der J. G. Cotta'schen Buchhandlung.

s. n. (s. a.): Redensarten.net. Damit Sie wissen, was Sie reden! www.redensarten.net [12.01.14].

Taylor, John R. (2007): Gramatyka kognitywna. (aus dem Engl. übersetzt von M. Buchta, Ł. Wiraszka). Kraków: Universitas.

Ziem, Alexander (2014): Kognitive Linguistik heute - Überblick über zentrale Positionen und Konzepte. www.phil-fak.uni-duesseldorf.de/fileadmin/Redaktion/Institute/Germanistik/ AbteilungI/Mitarbeiter/ziem/Ziem_KognitiveLinguistikHeute.pdf [02.03.14].

\footnotetext{
14 "Oba te znaczenia współistnieją i współgrają ze sobą tak w umyśle nadawcy, jak zapewne odbiorcy wypowiedzi. Obserwacje frazematyczne dowodzą, że taka 'dwutorowość znaczeniowa' jest realną rzeczywistością naszej komunikacji codziennej. Ów swoisty 'stan oscylacji semantycznej' między biegunami dosłowności i przenośności frazematyka uważa za naturalny i istniejący obiektywnie."
} 